\title{
An Update to the Present Carpal Tunnel Syndrome (CTS) Nerve Conduction Grading Tool
}

\author{
Salim Hirani* \\ Chief Clinical Physiologist, Department of Neurophysiology, Gwynedd Hospital, UK
}

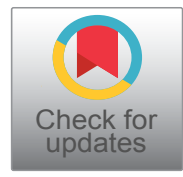

*Corresponding author: Salim Hirani, Chief Clinical Physiologist, Department of Neurophysiology, Gwynedd Hospital, Bangor, North Wales, UK

\begin{abstract}
The severity of carpal tunnel syndrome (CTS) may be categorised in a number of ways and utilising one of a range of presently available grading tools. The grading systems proposed by Bland and Padua, et al. are most commonly used. However, both are not without limitations, which are discussed comprehensively in this paper.

The aim of this research is to establish, using best available evidence, a clinically appropriate revision of the current CTS nerve conduction grading tool and to evaluate its effectiveness (in terms of acceptability and usability as a tool for intervention prediction). The revised scale is designed from a clinical physiology perspective and is based on numerical values of nerve conduction findings.

In contrast to Bland's grading of 1 to 6 , the proposed grading system is based on more nuanced descriptive categories, ranging from Normal to Early, Mild Sensory, Mild Sensory Motor, Moderate Sensory, Moderate Sensory Motor, Severe Sensory Motor, Extremely Severe Sensory Motor, and Complete Absence (Gradings 1-8). An additional two categories of clinical grading are therefore proposed.
\end{abstract}

\section{Keywords}

CTS grading tools, Neurophysiological grading tools for CTS, Carpal tunnel syndrome grading tool.

\section{Introduction}

The pathology of Carpal Tunnel Syndrome (CTS) is described as 'a neuropathy caused by entrapment of the median nerve at the level of the carpal tunnel' [1]. Nerve Conduction Studies (NCS) are one of the basic tools used to support the clinical diagnosis. NCS are objective tests that assess the physiological status of the median nerve across the carpal tunnel [2].

\section{Reasons for Grading Carpal Tunnel Syndrome}

The grading tool is used for electro-diagnostic as- sessment of CTS, in conjunction with the patient's clinical history and symptoms in order to diagnose the severity of CTS [3]. NCS results could influence the course of treatment and could enable the consultant to decide on the appropriate course of treatment for patient.

There are several primary tests performed as a first instance by the primary physician mentioned in different literature, including Phalen's, Tinel's and Durkan's signs which are subjective and are based on patient response. Other tests like Ultrasound, Nerve Conduction and Electromyogram (EMG) needle examination have all been used for CTS grading which are evidence base and not depending on the patient's clinical response [4].

However, to ascertain the severity of CTS, neurophysiological grading is required [5]. There are several grading scales specifically related to CTS; [6-10]. Most of the studies show grading in subjectivity. Some lack a Neurophysiological focus in objectivity, where the research was conducted on the basis of the primary tests only which was mentioned above $[3,8]$ during the collection of the data. Some researchers only use either Sensory Nerve Conduction Studies (NCS) or Motor NCS to differentiate the severity of CTS grading [7]. Not all the researchers have used sensitive techniques to diagnose early CTS or in severe cases, lumbricals responses to differentiate its severity from complete absence, which therefore cannot be diagnosed as CTS with complete certainty [11]. Other researchers lack robustness of data collection that could result toward biasness of the whole population [12].

It appears that whilst there is an accepted domi-

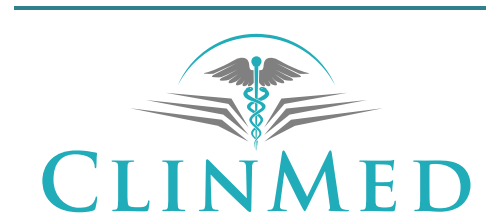

Citation: Hirani S (2020) An Update to the Present Carpal Tunnel Syndrome (CTS) Nerve Conduction Grading Tool. Int Arch Clin Physiol 2:006. doi.org/10.23937/iacph-2017/1710006

Accepted: May 18, 2020: Published: May 20, 2020

Copyright: (C) 2020 Hirani S. This is an open-access article distributed under the terms of the Creative Commons Attribution License, which permits unrestricted use, distribution, and reproduction in any I NTER NATIONAL LIBRARY medium, provided the original author and source are credited. 
nance of both the Bland and Padua, et al. $[3,5]$ grading systems, there are also clear limitations, which are discussed comprehensively in this paper.

In 2014 the Association of Neurophysiological Scientists (ANS), in collaboration with the British Society for Clinical Neurophysiology (BSCN) published guidelines outlining the accepted grading of CTS in the United Kingdom.

The aim of this research was to establish an evidence-based revision of the current CTS nerve conduction Grading Tool practice in the UK and to evaluate its effectiveness - in terms of acceptability as a tool for intervention prediction. This could enable the Surgeon to ascertain the level of severity and could help to decide on a conservative or surgical approach to treatment. Surgeon has to take their own decision for the treatment of CTS. A numerical value is given to each of the grade bandings to enable objective reporting and comparison [5].

No clinical assessment was conducted during or prior to conducting the NCS due to no Neurophysiologist was available.

\section{Methodology}

Ethical approval for the research project was obtained from the Heath Research Authority National Research Ethics Service London - Queen Square Research Ethics Committee (Reference 17/LO/0750).

Neurophysiological data was collected on the basis of the extensive and complete description of previous study designs by Padua and Canterbury $[3,5]$ and which is understood to be followed by most of the clinical laboratories in the United Kingdom as mentioned above paragraph. In addition, Second Lumbrical-Interosseous latency was also recorded to distinguish between 'very severe' and 'complete absent response' grading of CTS [13].

The test was performed by a qualified clinical physiologist (Neurophysiology) using Keypoint 9033A07 (Skovlunde, Denmark) machine, on the basis of the Neurophysiological departmental protocol, Bangor which was attached in appendix (Peripheral protocol, 2015). A quantitative method was used for collecting data [11], to ensure accuracy and to avoid bias as quantitative method based on numeric values. The sample size of patients in the study was use for all those tested for NCS over a period of one calendar year (2017), across the population of North Wales. During data collection, patients aged appears18 years and above, who were referred to the Neurophysiology department from the Orthopaedic and Neurology departments within the local Health Board, and General Practices (GPs) in North Wales. The inclusion criteria were based on the referral diagnosis; referral of CTS was considered based on paraesthesia, pain, swelling in median distribution area or digits I-V, worsened by sleep or driving or pins and needles, numbness, tingling or burning in all fingers. Exclusion criteria includes, radiculopathy, traumatic injury at the wrist, Ulnar nerve entrapment at wrist, Guyon's canal entrapment or dorsal ulnar coetaneous nerve injury at wrist.

Data was analysed on certain widely accepted assumptions of sensory amplitude and CV and distal motor latency (DML), amplitude and CV $[3,4]$. To introduce the term "mild", "moderate" and "severe", a numerical value was used during collection of data to differentiate the term which is accepted widely, and could be used to compare with other studies [5].

Sensory testing was performed first, by placing the stimulating ring electrodes on digit III, which is more sensitive then digit II, according to Bland, and the recording electrode over the median nerve at the wrist. The orthodromic technique was used for both sensory and motor Nerve Conduction Studies (NCS) test, for median and ulnar nerves. A maximal current was applied to record the full response of the nerve, at the digits II-IV for median sensory and digit $\mathrm{V}$ for ulnar sensory recording. Stimulating median nerve pathways at the wrist and at the elbow for motor nerves, recording median nerve responses from abductor polices brevis (APB), and ulnar nerve pathways from first dorsal interosseous (FDI). Digit II was tested only when the response from digit III was less than $3 \mu \mathrm{V}$ or absent; digit IV stimulated only when the response from digit III showed a conduction velocity between $45-50 \mathrm{~m} / \mathrm{sec}$. Amplitude was recorded peak to peak for sensory responses, and base to peak for motor responses. If responses were not recordable from median sensory and motor nerves at the APB muscle, then the responses were elicited by placing recording electrodes on the $2^{\text {nd }}$ lumbricals, stimulating median and ulnar nerves at the wrist $[8,13,14]$ (photos attached in appendix).

All patient data was collected by fulfilling the criteria mentioned in above paragraph depending on the severity. The reason for using the new criteria is to describe the full range of severity which was not fully covered by other researchers mentioned earlier in this paper. Criteria was mentioned in above paragraph are intended to be reliable in terms of grading. Propose gradings are as follows.

Normal (Grade 0): Where sensory conduction velocity SCV from digit III is above $50 \mathrm{~m} / \mathrm{s}$ and amplitude $\geq 5$ $\mu \mathrm{V}$, with distal motor latency (DML) $\leq 4.2 \mathrm{~ms}$, amplitude $\geq 5 \mathrm{mV}$, and conduction velocity $(\mathrm{CV}) \geq 50 \mathrm{~m} / \mathrm{s}$.

Early (Grade 1): Where SCV is between $45-50 \mathrm{~m} / \mathrm{s}$ from digit III, and double peak latency in digit IV is $>0.5$ $\mathrm{ms}$, with $\mathrm{DML} \leq 4$ and normal sensory and motor amplitudes $>5$ - sensory in $\mu \mathrm{V}$; motor in $\mathrm{mV}$.

Mild Sensory (Grade 2): Where SCV is between 40$44.9 \mathrm{~m} / \mathrm{s}$ from digit III, with normal sensory amplitude and motor values. 
Mild Sensory-Motor (Grade 3): Where SCV is between $40-44.9 \mathrm{~m} / \mathrm{s}$ from digit III with normal sensory amplitude, $\mathrm{DML} \geq 4.2 \mathrm{~ms}$, with normal motor amplitude and CV.

Moderate Sensory (Grade 4): Where SCV is less than $40 \mathrm{~m} / \mathrm{s}$ from digit III, with normal sensory amplitude and normal motor values.

Moderate Sensory-Motor (Grade 5): Where SCV is less than $40 \mathrm{~m} / \mathrm{s}$ from digit III, with normal sensory amplitude, $\mathrm{DML} \geq 4.2 \mathrm{~ms}$, with normal motor amplitude and CV.

Severe Sensory-Motor (Grade 6): Where sensory potentials from digits III and digit II are absent or $<3$ $\mu \mathrm{V}$ in both digits III and II, with SCV $<30 \mathrm{~m} / \mathrm{s}, \mathrm{DML} \geq 4$. , MCV either slow or normal.

Extremely Severe Sensory-Motor (Grade 7): Where sensory and motor potentials are absent and response recordable only from $2^{\text {nd }}$ lumbricals, where median lumbricals are prolonged compared to ulnar lumbricals.

Complete (Grade 8): Where both sensory and motor potentials are absent and responses are not recordable from median $2^{\text {nd }}$ lumbricals, but recordable from ulnar $2^{\text {nd }}$ lumbricals (please refer to the comparison of Bland grading with the proposed revised grading, Table 1, for clarification).

\section{Results}

The data was collected for a period of one year (2017). Initially a total of 1132 patients were included in this study. Both hands were tested (symptomatic and asymptomatic). During data collection, two referrals were not included, because the patients declined to participate in all the study procedures; seven participants' data sets were excluded from the analysis because the departmental protocol for the peripheral nerve conduction study was not followed. Therefore, 1123 patients (2246) hands were included in the final data collection.

Of the 1123 patients, 687 were female and 436 were male. The age range was 19 to 98 years, median age 56 years. The numbers of hands in each grade of severity are shown in Figure 1 and Table 2 - Grading vs. number of hands.

\section{Discussion}

Bland, recorded that prolongation of motor terminal latency to APB is not significant in mild cases which I

Table 1: Values comparison with grading of Padua, Bland and Hirani.

\begin{tabular}{|c|c|c|}
\hline Padua [5] (\%) & Bland [3] (\%) & Hirani (\%) \\
\hline Normal -18 (3) & Normal - 3629 (42.5) & Normal - 968 (43) \\
\hline Minimal - $123(20.5)$ & Very mild - 684 (8) & Early - 271 (12) \\
\hline \multirow[t]{2}{*}{ Mild - $145(24)$} & Mild sensory-motor - 944 (11) & Mild sensory - 215 (10) \\
\hline & & Mild S/M - 51 (2) \\
\hline \multirow[t]{2}{*}{ Moderate - $217(36)$} & Moderately Severe - 1359 (16) & Moderate sensory - $134(6)$ \\
\hline & & Moderate S/M - 356 (16) \\
\hline Severe - 81 (13.5) & Severe - $568(7)$ & Severe S/M - 204 (9) \\
\hline \multirow[t]{2}{*}{ Extremely severe - $16(3)$} & Very severe - $930(11)$ & Extremely Severe S/M - 33 (1) \\
\hline & Extremely Severe - 387 (4.5) & Complete - 14 (1) \\
\hline Total Hands: 600 & 8501 & 2246 \\
\hline
\end{tabular}

\section{CTS Gradiings}

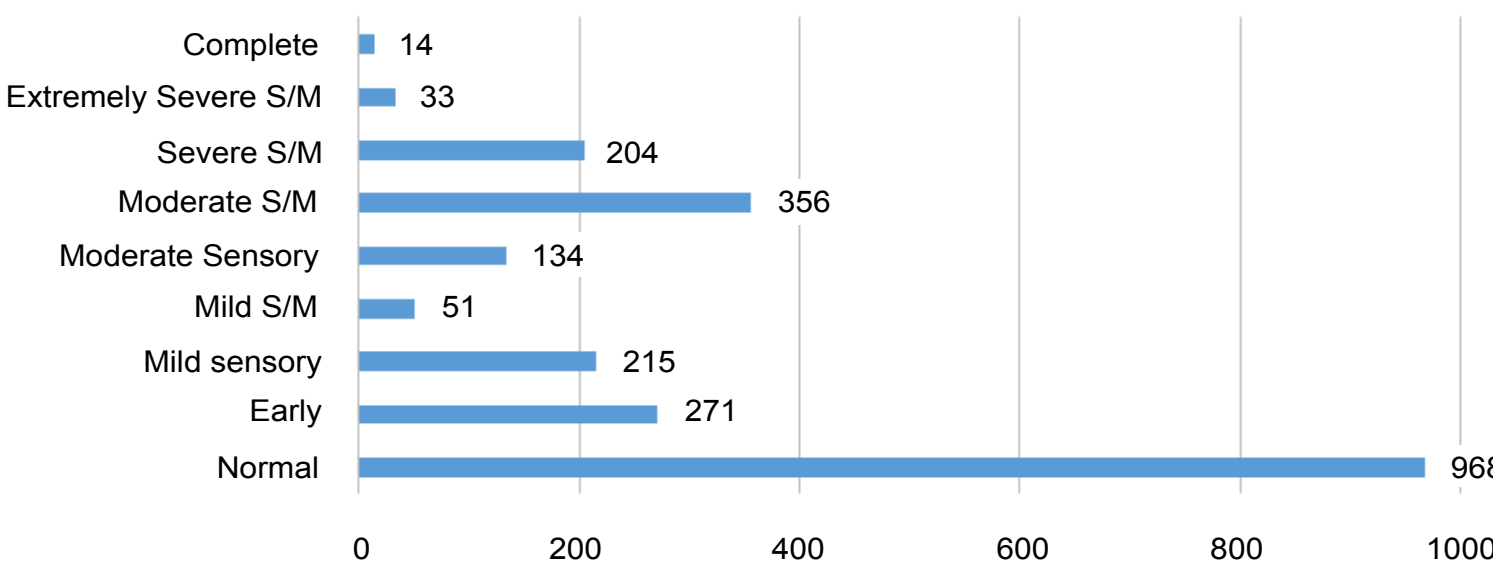

Figure 1: Grading vs. number of hands. 
Table 2: Grading vs. number of hands

\begin{tabular}{|l|l|}
\hline Grading & Number of hands \\
\hline Normal & 968 \\
\hline Early & 271 \\
\hline Mild sensory & 215 \\
\hline Mild S/M & 51 \\
\hline Moderate Sensory & 134 \\
\hline Moderate S/M & 356 \\
\hline Server S/M & 204 \\
\hline Extremely Server S/M & 33 \\
\hline Complete & 14 \\
\hline Total Hands & 2,246 \\
\hline
\end{tabular}

partially agree with, as it appears insignificant in above graph as compared to mild sensory CTS [3]. It was noted that both Padua, et al., and Bland [3,5] didn't separate the mild sensory and mild sensory-motor in their Grading. This clearly indicates that there is a utility for some revision and a separate grading in mild CTS. However, when compared to 'moderate sensory and motor CTS' with moderate sensory CTS, moderate sensory-motor CTS has significantly higher cases than the moderate sensory. This clearly indicates that there is a utility for some revision and a separate grading in moderate CTS.

The Bland grading system enables the neurophysiologist to differentiate levels of severity, whilst providing the surgeon clear clinical reasoning data, which would ascertain the appropriate treatment to follow - conservative or surgical and thus, enabling a prognosis predictor for the patient [5]. However, due to the limited numerical grading in 5 and 6 , author felt that the Bland grading does not enable the level of severity to be objectively and fully as curtained as possible.

In theory, the higher the grade, the greater the nerve dysfunction [3]. But the analysis of data in this study appears to show mixed levels of severity. A majority of CTS studies in this investigation appear normal, due to the fact that non-symptomatic hand were also recorded, to fulfil department protocol. Although normal (Grade 0 ) has much higher numbers compare to other grades, this does not detract from the fact that levels of severity for CTS were found in different grade. In this study, comparison of the grading scales shows that there are not big differences in the "mild" group between Bland's and my groups, with $11 \%$ in Bland's mild sensory-motor category with $10 \%$ mild sensory and only $2 \%$ mild mixed sensory motor, which might suggest that the separation is marked, although the separation has value in demonstrating the effects in the motor fascicles [3]. However at the moderate degree of severity, there is a notable difference with $16 \%$ in Bland's moderate category and $6 \%$ moderate sensory and $16 \%$ moderate mixed sensory motor, suggestive that these are 2 groups worth separating [3].
Padua, et al. relates the outcome of grading with surgical decompression, compared to Bland's grading, which is based purely on neurophysiological concepts. Bland's grading scale was based on a very large population. However, in the 'severe' grade and 'extremely severe' grade, his values are not clear. He has given the amplitude for the motor response, but has not taken into account the DML and CV. And, for 'extremely severe' grading, although the amplitude in the motor CMAP has been taken into account, no account has been taken of distal latency and CV $[3,5]$.

Comparing the Bland grading system of CTS with the grading suggested in this paper, it seems that Bland Grades 0 and 1 are comparable with our proposed grading. I would suggest that the Bland grading for grade 2 needs to be more elaborate, by dividing into two separate grades, i.e. mild sensory and mild sensori-motor, as sensory is more significant, compared to sensory and motor together. In the revised grading the sensory involvement is graded as Grade 2, and the sensori-motor where both functions are involved is graded as Grade 3 [3].

Bland covers a moderate degree of severity in grade 3 , which again warrants further elaboration to make the gradings more objective and more descriptive. In my revised system, grade 4 covers sensory involvement and grade 5 covers both sensory and motor involvement. Bland's grade 4, which is severe CTS, compares favourably with grade 6 of the revised grading system [3].

Bland only describes a prolonged DML in grade 5 as very severe CTS, which I believe does not fulfil all criteria to separate from his grade 6 . He appears not to have taken any account of changes in sensory potentials or motor conduction velocity values. The revised grading system has graded Very Severe CTS where both sensory and motor responses are absent and responses were only recordable from the $2^{\text {nd }}$ lumbricals, with prolonged median distal latency compared to ulnar lumbricals as Grade 7 [3].

The grade 6 in Bland again has the potential to create confusion, as it refers to a low amplitude motor potential. It appears not to have taken into account the distal motor latency. Bland also not included CV in Grade 5. The author has considered the latency and amplitude with $\mathrm{CV}$ and graded as complete median nerve dysfunctions where both median motor and sensory as well as median $2^{\text {nd }}$ lumbrical responses are absent and the only response appears in the ulnar $2^{\text {nd }}$ lumbrical. This grade appears as grade 8 in the proposed grading system [3].

Table 3 summarizes and compares the Bland (2000) grading with the revised grading system.

\section{Conclusion}

The grading system devised by Bland and used to grade the levels of severity of CTS over the last 17 years 
Table 3: Comparison of Bland (Bland, 2000) grading with the authors proposed grading.

\begin{tabular}{|c|c|c|}
\hline Grading & Bland & Modified grading by Hirani \\
\hline Grade 1 & $\begin{array}{l}\text { Inching, palm/wrist median/ } \\
\text { ulnar comparison, ring finger } \\
\text { double peak }\end{array}$ & $\begin{array}{l}\text { Early: } \\
\text { SCV }=45-50 \mathrm{~m} / \mathrm{s} \text { interpeak potentials in digit IV }>0.5 \mathrm{~ms} \text {, Distal Motor Latency } \\
(\mathrm{DML})<4.2 \mathrm{~ms} \text {. Amplitude of sensory and motor potentials } \geq 5\end{array}$ \\
\hline Grade 2 & $\begin{array}{l}\text { Mild Sensory: Sensory } \\
\text { conduction velocity (SCV }< \\
40 \mathrm{~m} / \mathrm{s} \text { DML }<4.5 \mathrm{~ms}\end{array}$ & $\begin{array}{l}\text { Mild sensory: } \\
\text { SCV }=40-44.9 \mathrm{~m} / \mathrm{s} \text { with normal sensory amplitude (NSA), DML, amplitude and CV }\end{array}$ \\
\hline Grade 3 & $\begin{array}{l}\text { Moderately Severe: DML } \\
>4.5 \text { ms and }<6.5 \mathrm{~ms} \\
\text { with Sensory nerve action } \\
\text { potentials (SNAP) preserved }\end{array}$ & $\begin{array}{l}\text { Mild sensory motor: } \\
\mathrm{SCV}=40-44.9 \mathrm{~m} / \mathrm{s} \text { with } \mathrm{NSA}, \mathrm{DML}>4.2 \mathrm{~ms} \text { with normal motor amplitude (NMA) } \\
\text { and normal } \mathrm{CV}(\mathrm{NCV})\end{array}$ \\
\hline Grade 4 & $\begin{array}{l}\text { Severe: } \mathrm{DML}>4.5 \mathrm{~ms} \text { and }< \\
6.5 \mathrm{~ms} \text { with absent SNAP }\end{array}$ & $\begin{array}{l}\text { Moderate sensory: } \\
\text { SCV }<40 \mathrm{~m} / \mathrm{s} \text { with NSA, normal DML, NMA and NCV }\end{array}$ \\
\hline Grade 5 & Very Severe: DML > 6.5 ms. & $\begin{array}{l}\text { Moderate sensory motor: } \\
\mathrm{SCV}<40 \mathrm{~m} / \mathrm{s} \text { with } \mathrm{NSA}, \mathrm{DML}>4.2 \mathrm{~ms} \text { and MNA and NCV }\end{array}$ \\
\hline Grade 6 & $\begin{array}{l}\text { Extremely Severe : Motor } \\
\text { nerve action potentials } \\
(\text { MNAP })<0.2 \mathrm{mV}\end{array}$ & $\begin{array}{l}\text { Severe Sensory motor: } \\
\text { Absent or }<3 \mu \mathrm{V} \text { SNAP with SCV }<30 \mathrm{~m} / \mathrm{s} \text { and } \mathrm{DML}>4.2 \mathrm{~ms} \text { with either slow or } \\
\text { normal MCV and or NMA }\end{array}$ \\
\hline Grade 7 & & $\begin{array}{l}\text { Extremely severe: } \\
\text { SNAP and CMAP absent, but recordable from both median and ulnar } 2^{\text {nd }} \\
\text { lumbricals with prolonged median } 2^{\text {nd }} \text { lumbrical response compared to ulnar } \\
\text { lumbricals }\end{array}$ \\
\hline Grade 8 & & $\begin{array}{l}\text { Complete: } \\
\text { SNAP and CMAP absent and absent median } 2^{\text {nd }} \text { lumbrical and present ulnar } 2^{\text {nd }} \\
\text { lumbrical. }\end{array}$ \\
\hline
\end{tabular}

in most Neurophysiology clinic in the UK has certain limitations, and the author believes system needs modification in order to accommodate current practice [3]. Propose grading system for CTS is based on a review of a current and past literature and also based on data collection from entire North Wales.

Bland and Padua, et al. both limited the DML and $\mathrm{CV}$ in motor study and amplitude potentials and CV in sensory study. Author follow the same rule and precede the study with below given cut off values to grade them accordingly. Many Clinical laboratories in UK use the above criteria of cut off values for sensory and motor study and follow either Bland or Padua at al. [3,5]. Presently, there is no standard of CTS grading followed throughout the UK due to their limitations. Proposed grading scale preferably was felt to be an acceptable tool for Clinical Physiologists due to its coverage.

The revised grading tool using a physiological basis offers a more precise numerical grading, which is both objective and repeatable. This will enable the surgeon to precisely ascertain the level of severity and decide on a conservative or surgical approach to treatment. Surgeons have to take their own decision for the treatment of CTS. Surgeons could consider proposed Grade 1-3 for conservative treatment and Grade 4-6 for surgical intervention where the chances of full recovery from CTS syndrome due to APB muscles still intact. Surgeon has to think for surgical intervention for Grade 7-8 cases whether it would be beneficial or not for presence of APB fibres, age and other medical history of the patient. Future studies looking at prognosis may be helpful in looking at the outcomes from different interventions for those with different gradings of severity and to look at the implications of motor involvement compared with just sensory fascicle involvement.

\section{Acknowledgements}

The author would like to acknowledge and thank Mrs. Julie Evans, Dr. Gareth Payne, and Dr. Bashir Kassam for their encouragement, guidance and help with this study.

\section{References}

1. Ihsan M Ajeena, Raed H Al-Saad, Ahmed Al-Mudhafar, Najah R Hadi, Awsan H Al-Aridhy (2013) Ultrasonic assessment of females with carpal tunnel syndrome proved by nerve conduction study. Neural Plast 2013: 754564.

2. Giannini F, Cioni R, Mondelli M, Padua R, Gregori B, et al. (2002) A new clinical scale of carpal tunnel syndrome: Validation of the measurement and clinical-neurophysiological assesment. Clin Neurophysiol 113: 71-77.

3. Bland J (2000) A neurophysiological grading scale for carpal tunnel syndrome. Muscle Nerve 23: 1280-1283.

4. Alfonso C, Jann S, Massa R, Torreggiani A (2010) Diagnosis, treatment and follow-up of the carpal tunnel syndrome: A review. Neurol Sci 31: 243-252.

5. Padua L, Monaco ML, Valente EH, Tonali PA (1996) A useful electrophysiologic parameter for diagnosis of carpal tun- 
nel syndrome. Muscle Nerve 19: 48-53.

6. Campbell EDR (1962) The carpal tunnel syndrome: Investigation and assessment of treatment. Proc R Soc Med 55: 401-405.

7. Leventoglu A, Kuruoglu R (2006) Do electrophysiological findings differ according to the clinical severity of carpal tunnel syndrome? Neurological Sciences (Turkish) 23: 272278.

8. Carvalho FN, Carneiro AP, Paulinelli RR, Carvelho TN (2007) Neurophysiological classification of the carpal tunnel syndrome. Acta Fisiatrica 14: 190-195.

9. Jeong DH, Kim CH (2014) The quantitative relationship between physical examination and the nerve conduction of the carpal tunnel syndrome in patients with and without a diabetic polyneuropathy. Ann Rehabil Med 38: 57-63.
10. Jerosh-Herold C, Shepstone L, Wilson ECF, Dyer T, Blake $\mathrm{J}$ (2014) Clinical course, costs and predictive factors for response to treatment in carpal tunnel syndrome: The PALMS study protocol. BMC Musculoskelet Disord 15: 35.

11. AAEM, AAN, AAPMR (1993) Practice parameter for electrodiagnostic studies in carpal tunnel syndrome: Summary statement. Muscle Nerve 16: 1390-1391.

12. Ibrahim I, Khan WS, Goddard N, Smitham P (2012) Carpal tunnel syndrome: A review of the recent literature. Open Orthop J 6: 69-76.

13. Ozben S, Acar H, Gunaydin S, Genc F, Ozer F, et al. (2012) The second lumbrical-interosseous latency comparision in carpal tunnel syndrome. J Clin Neurophysiol 29: 263-267.

14. Preston D, Logigian EL (1992) Lumbrical and interossei recording in carpal tunnel syndrome. Muscle Nerve 15: 12531257. 